\title{
ANOMALÍAS DENTARIAS DE NÚMERO, PRESENTES EN PACIENTES CON LABIO Y PALADAR FISURADO, DE 6 A 12 AÑOS DE EDAD. REVISIÓN DE LITERATURA
}

\section{Dental anomalies of number, present in patients with cleft lip and palate, 6 to 12 years of age. Literature review.}

\begin{abstract}
Mayancela Mayancela Jorge ${ }^{1}$, Alvarado Cordero Alberto ${ }^{2}$
${ }^{1}$ Estudiante de Pregrado de la Universidad Católica de Cuenca. Cuenca, Ecuador. 2 Especialista en Ortodoncia y Ortopédia Maxilo Facial, Docente de la Carrera de Odontología, Universidad Católica de Cuenca. Ecuador.
\end{abstract} DOI: https://doi.org/10.31984/oactiva.v6i3.620

\section{Resumen}

La Fisura de labio y paladar (LPF), es una malformación cráneo-facial congénita; que provoca alteraciones estéticas y funcionales a nivel bucofacial, lo cual origina problemas de alimentación, fonación, audición, entre otros. Uno de los grandes problemas presentes en estos pacientes son las alteraciones a nivel de los órganos dentarios de los cuales destacan las anomalías dentarias de número reportados como las más prevalentes en pacientes con LPF. Objetivo: Obtener información sistematizada y actualizada referente a las anomalías dentarias de número (agenesia y dientes supernumerarios), presentes en pacientes con LPF de 6 a 12 años de edad. Discusión: La literatura reporta una evidente prevalencia mayoritaria de anomalías dentales en pacientes con LPF, que en la población en general, autores como Renfigo H. Vigueras O. De Stefani A; entre otros, coinciden en sus investigaciones, mencionando que las principales anomalías dentales presentes en pacientes con LPF son las agenesias y dientes supernumerarios, (anomalías dentarias de número). Conclusión: La agenesia dental ocupa la anomalía dental más prevalente en individuos con labio y paladar fisurado, seguida del diente supernumerario, estas dos alteraciones dentales de acuerdo a diferentes estudios originales ocupan los primeros puestos en cuanto a prevalencia.

Palabras clave: Anomalías dentarias; diente supernumerario; agenesia dental; labio hendido; fisura del Paladar; Labio y Paladar hendido.

\begin{abstract}
Cleft lip and palate (CLP) is a congenital craniofacial malformation that causes aesthetic and functional alterations at the oral-facial level, which causes problems in feeding, phonation, hearing, among others. One of the major problems are the alterations at the level of the dental organs, of which the most prevalent in patients with CLP are the dental anomalies of number. Objective: To obtain systematized and updated information on dental anomalies of number (agenesis and supernumerary teeth), present in patients with CLP from 6 to 12 years of age. Discussion: The literature reports an evident higher prevalence of dental anomalies in patients with CLP than in the general population. Authors such as Renfigo H., Vigueras O., De Stefan A., among others, agree in their research that the main dental anomalies present in patients with CLP are agenesis and supernumerary teeth (dental anomalies of number). Conclusion: Dental agenesis is the most prevalent dental anomaly in individuals with cleft lip and palate, followed by the supernumerary tooth, these two dental alterations according to different original studies occupy the first positions in terms of prevalence.
\end{abstract}

Key words: Dental anomalies; Tooth Supernumerary; Tooth agenesis; Cleft Lip; Cleft Palate; Cleft Lip and palate.

\section{Introducción}

La Fisura de labio y paladar (LPF), es una malformación cráneo-facial congénita; que se manifiesta con gran frecuencia en la población a nivel mundial, 1 de cada 1000 nacidos vivos presentan esta alteración. ${ }^{1}$ Países Latinoamericanos como: Bolivia, Ecuador y Paraguay muestran las tasas más altas de prevalencia en esta región.1,2,3 Esta malformación puede relacionarse o ser parte de un síndro- me o no serlo (LPF no sindrómica), y se afirma que el 70\% de las Fisuras Labio Palatinas, no se relacionan a ningún síndrome. ${ }^{1,3}$

La Fisura Labio Palatina, también llamada Hendidura Orofacial, se encuentra como una de las patologías más frecuentes que altera las estructuras bucofaciales, ${ }^{4,5}$ causando un impacto negativo a nivel social, psicológico, estético y funcional del paciente afectado. ${ }^{1,4}$ Problemas 
como la alimentación, fonación, audición, alteraciones dentales, entre otras son los desafíos a los que se enfrenta el paciente y su familia. ${ }^{2,4}$ Debido a la variedad de alteraciones que se manifiesta en el paciente, diversos especialistas deben trabajar en conjunto, de tal manera, que su tratamiento sea multidisciplinario para lograr resultados enfocados en mejorar la calidad de vida. ${ }^{1,5}$

Según Palacios D. en $2020^{5}$, en su artículo señala, “El tratamiento de pacientes con LPF ha sido definido como una empresa multidisciplinaria e interdisciplinaria, comenzando el tratamiento desde una etapa temprana o prenatal, el mismo que continua a lo largo de la vida del paciente". 5 Dentro del equipo interdisciplinario, juega un papel importante las actividades del odontólogo, que inician desde un diagnóstico temprano y una orientación de los diferentes tratamientos dentales hasta lograr un completo bienestar del paciente desde el punto de vista odontológico; pues es el Odontólogo el responsable de la salud bucal del paciente, siempre interactuando con las diferentes especialidades, para brindar un tratamiento integral. ${ }^{1,5,6}$

Entre los roles que cumple el odontólogo, está el reconocer las diferentes anomalías dentales que se manifiestan en estos pacientes, entre ellas se encuentran las alteraciones dentales de forma, tamaño, número, posición, erupción, entre otras. ${ }^{1,7}$ Según investigaciones científicas las alteraciones dentales que mayor prevalencia muestran son las anomalías de número. ${ }^{7}$ Autores como Renfigo $\mathrm{H}$. (2015), Vigueras O. (2015), De Stefani A. (2019), entre otros, coinciden en sus investigaciones, que las principales anomalías dentales presentes en pacientes con LPF son la agenesia y dientes supernumerarios, (anomalías dentarias de número). ${ }^{1,7}$

Se señala que la agenesia dental puede manifiestarse en un $40 \%$ en pacientes con LPF; y en un $28 \%$ dientes supernumerarios, esto de acuerdo a una investigación realizada por Sanchez P. (2019). ${ }^{1}$

Desde este punto de vista, debido a la alta prevalencia de las alteraciones dentales que se presenta en estos pacientes, nos hemos planteado realizar esta revisión bibliográfica con el objetivo de proporcionar información sistematizada y actualizada sobre las anomalías dentales de número y así brindar información relevante, para estudiantes y profesionales de la salud.

\section{Estado del Arte}

La siguiente investigación es una revisión de la literatura científica publicada en los últimos 5 años, en el periodo 2015 - 2020. La búsqueda bibliográfica se realizó en las bases de datos de Scopus, PudMed, EBSCO, Scielo, Google Académico, Redalyc. Los términos (palabras claves), utilizados para la búsqueda de información fueron aquellos que constan en los Descriptores de Ciencias de la salud (DeCS) y Medical Subject Headings (MeSH), entre estas mencionamos: "Anomalías Dentarias", "Diente supernumerario", "Agenesia Dental", "Labio Hendido", "Fisura del Paladar", "Labio y Paladar Hendido". Se seleccionaron los artículos en función del título, contenido y año de publicación, se omitieron aquellos que no mostraban relevancia, obteniendo un total de 34 referencias bibliográficas.

Los artículos seleccionados se centraban en temas como conceptos, etiología, prevalencia, diagnóstico y tratamiento del Labio y Paladar Fisurado (LPF), además se seleccionaron aquellos estudios originales, los cuales evaluaron anomalías dentarias en individuos con LPF.

\subsection{Aspectos generales:}

La presencia de Labio y Paladar Fisurado (LPF), comúnmente llamada Hendidura del Labio y Paladar o denominado en varios artículos como Fisura Labio Alveolo Palatina (FLAP), son malformaciones que se manifiestan debido a una falla en la fusión de las estructuras craneofaciales, en la etapa de gestación o vida intrauterina. ${ }^{3,6}$

Embriológicamente, en el inicio de la cuarta hasta la octava semana de embarazo, es cuando se produce esta malformación, debido a una alteración en la fusión parcial o completa de las cinco prominencias faciales, (la prominencia frontonasal, dos prominencias maxilares y dos mandibulares), las cuales darán origen a estructuras anatómicas como el labio superior y el paladar. ${ }^{3,6}$

La prominencia frontonasal se origina a partir de una proliferación de la mesénquima, ubicada sobre el estomodeo, formando así estructuras anatómicas como: la mitad superior de la cara, filtrum labial, el septum nasal, premaxila y el paladar duro anterior; ${ }^{3}$ mientras que las prominencias o también llamados procesos maxilares formarán la mitad superior de las mejillas, las porciones laterales del labio superior y el paladar posterior, finalmente las prominencias (procesos) mandibulares formarán: la mandíbula, la mitad inferior de las mejillas y parte de la lengua. ${ }^{3}$

Según las estructuras anatómicas afectadas y la severidad de éstas, se han establecido diferentes clasificaciones, debido a que la Fisura Labio Palatina se puede manifestar de varias maneras, ya sea de forma aislada, combinada, unilateral o bilateral. ${ }^{4,6}$

A partir de estas definiciones, a través del tiempo han existido varias propuestas para clasificar cada una de estas entidades, por parte de varios autores, quienes han tratado de establecer una clasificación que sea entendible, fácil y útil en la práctica clínica. ${ }^{3}$ 
En un inicio la Fisura de Labio y Paladar ha sido clasificada de la siguiente manera: Labio y Paladar Fisurado (LPF), Labio Fisurado (LF), Paladar Fisurado (PF), ya sean uni o bilaterales, de forma aislada o asociada. Esta es una de las clasificaciones más sencillas y prácticas aceptado a nivel mundial, establecida por Davis y Ritchie (1922). ${ }^{6,9}$

En relación con la etiología, hasta la actualidad no ha sido completamente establecida, sin embargo, se sabe que su origen es multifactorial y abarca diferentes factores ambientales, teratógenos y genéticos. ${ }^{3,10}$ Según la literatura factores ambientales como la exposición a pesticidas, ingesta de alcohol, edad materna, infecciones durante la gestación, contacto con radiaciones, desnutrición, consumo de tabaco, plomo entre otras, a las que las mujeres se exponen en la etapa pregestacional y durante las primeras semanas de gestación son factores de importancia para que se presente esta alteración. ${ }^{1,2,4}$

Sánchez M. (2019)1 señala que "El 25\% de los casos de LPF se conoce la causa, de los cuales el $75 \%$ de los casos es multifactorial y del $20 \%$ al $25 \%$ existe algún antecedente familiar".

Martínez L. (2018) ${ }^{4}$ menciona que "El 70\% de los casos de LPF está ligada a factores ambientales, y el $30 \%$ se relaciona a antecedentes familiares; además, el mismo autor afirma que uno de cada cinco pacientes en diferentes poblaciones tiene una historia familiar positiva."

\subsection{Prevalencia del Labio y Paladar Fisurado}

La prevalencia del Labio y Paladar Fisurado es significativa a nivel mundial, según varias investigaciones definen a esta malformación como una de las más frecuentes y predominantes; pues se estima que la prevalencia a nivel mundial se ve en 1 de cada 700-1000 nacidos vivos. ${ }^{1,4}$ Adicional a esto su prevalencia dependerá sustancialmente de las diferentes zonas geográficas y grupos étnicos; ${ }^{1}$ así mismo se debe mencionar los casos que están relacionados con síndromes, los cuales constituyen un $30 \%{ }^{1,11}$

Según investigaciones realizados en diferentes partes del mundo señalan diferentes prevalencias de acuerdo a la zona geográfica investigada, es así que el Labio y Paladar Fisurado en la población asiática se manifiesta en 1-500 nacidos vivos, siendo en este lugar la más prevalente. En la población caucásica, hispanos y latinos se presenta en una prevalencia de 1 por cada 1000 nacidos vivos y en la raza negra se observa en 1 por cada 2500 nacidos vivos, siendo la menos prevalente. ${ }^{12}$

Países Asiáticos como China es donde se presenta la más alta prevalencia de fisuras labio palatina, donde la incidencia varía de 1.2 por cada 1000 a 30.7 por cada 10000 $(1.2-3.07 / 1000) .{ }^{11}$
Países Latinoamericanos como: Bolivia, Ecuador y Paraguay muestran las tasas más altas de prevalencia en esta región. ${ }^{1,2,3}$ Cazar M. en $2020^{2}$ menciona "El Centro para Estudios Médicos y el Instituto Latinoamericano de Malformaciones Congénitas, refiere que la tasa global en la región es de 10.49 por 10000 nacidos vivos". Por el contrario, países como Venezuela, Perú, Brasil muestran las tasas más bajas de prevalencia. ${ }^{2}$

Según un estudio realizado por Cazar M. en 2020.2. "En Ecuador la prevalencia se estima en un $2.9 \%$ a un $3.7 \%$. con una frecuencia de 14.97 por cada 10000 nacidos vivos, dato concordante con otros países de la región. Según registros hospitalarios del sistema nacional de salud, 1132 pacientes con diagnóstico LPF fueron atendidos en el periodo 2010 - 2018."

De acuerdo a la anatomía que presenta esta malformación, la literatura menciona que el $21 \%$ se presenta de forma aislada como Labio Fisurado (LF) y el 33\% como Fisura Palatina (PF), mientras que el $46 \%$ se presenta como una combinación de ambas formas (LPF). ${ }^{12}$ Además se menciona que el lado más afectado es el izquierdo y que los varones son más propensos a padecer esta patología. ${ }^{7}$

\subsection{Diagnóstico y tratamiento:}

El diagnóstico se basa en relación a las manifestaciones clínicas que se presentan al momento del nacimiento, debido a que su aspecto morfológico es evidente cuando el paciente es afectado por esta malformación, algunas de las características clínicas fenotípicas del paciente es la desviación del filtrum en relación al eje vertical de la cara del paciente, que se orienta a la narina afectada, además la punta de la nariz se desvía hacia el lado afectado. ${ }^{3}$

El diagnóstico prenatal se considera el aspecto más importante, y actualmente se cuentan con métodos de diagnóstico bastante confiables, por ejemplo, los estudios de imagen como la ecografía en tercera dimensión hace que el abordaje terapéutico sea más efectivo. ${ }^{3}$

En cuanto al tratamiento, varios artículos señalan la importancia de abordar una terapia donde distintas disciplinas trabajen en conjunto y coordinados, con el objetivo de brindar una atención integral al paciente, abordando desde los problemas más básicos hasta los más complejos.

Los pacientes que se ven afectados por esta alteración manifiestan un impacto negativo a nivel social, psicológico, estético y funcional. ${ }^{1,4}$ Problemas como la alimentación, fonación, audición y alteraciones dentales, entre otras, son los desafíos a los que se enfrenta el paciente y su família. ${ }^{2,4}$ 
Según Palacios D. (2020) "El tratamiento de pacientes con hendiduras orofaciales es una empresa multidisciplinaria e interdisciplinaria que ejerce sus funciones desde la etapa prenatal con el asesoramiento familiar y continúa desarrollando roles estratégicos que se extienden a lo largo de la vida del individuo. Reconocer que el manejo del paciente con Labio y Paladar Fisurado (LPF) es complejo, implica involucrar múltiples especialistas de la salud; cuya interacción es clave para estructurar un equipo de atención exitoso". Especialistas como el cirujano maxilofacial, fonoaudiólogo, odontopediatra, ortodoncista, ortopédico, psicólogo, entre otros, son algunos de los profesionales que deben ser parte del equipo interdisciplinario. ${ }^{1,5}$

\subsection{Anomalías dentarias en pacientes con Labio y Paladar Fisurado}

La presencia de anomalías dentales en pacientes con LPF es bastante frecuente, pues está bien documentada en la literatura que los niños afectados por esta alteración son notablemente más predisponentes a manifestar diferentes anomalías dentales en comparación con la población en general. ${ }^{13}$

Entre los diferentes tipos de anomalías dentales se encuentran: alteraciones de número, forma, tamaño, posición y erupción; las cuales afectan tanto a la dentición temporal como a la dentición permanente. ${ }^{14}$ Dichas alteraciones se manifiestan tanto en el área de la fisura, como fuera de ella y además puede manifestarse en la mandibula. ${ }^{13}$

Según un estudio realizado por Rengifo R. en $2016^{14}$ (Colombia) en una población de niños con Fisura Labio Palatina encontró que las anomalías más prevalentes fueron agenesia dental, mayor al $90 \%$ y dientes supernumerarios en un $40 \%$

Es así que dentro de las alteraciones dentales más comunes está la agenesia dental y dientes supernumerarios. según Rizell S. en 201913 "La agenesia se manifiesta en un $40 \%$ a $61 \%$ de los niños afectados con LPF"; y Navas M. en $2017^{15}$ manifestó que "Los dientes supernumerarios, reportan una prevalencia en pacientes con labio y paladar hendido en un $28 \%$ ".

\subsection{Agenesia dental en pacientes con LPF}

La agenesia dental definida como la ausencia congénita de uno o más órganos dentales ya sea temporales o permanentes, es una patología dental que se presenta con gran frecuencia en pacientes con Fisuras Labio Palatina, asociada con todos los tipos de fisuras. ${ }^{16,17}$

Aunque se reporte que la agenesia dental es muy prevalente en la población general y sea la anomalía dental de desarrollo más común notificada en humanos, estudios realizados señalan que su prevalencia en pacientes con LPF son 7 veces más frecuente. ${ }^{16,17}$

Berniczei A. en $2016^{17}$ menciona "En una investigación sobre la agenesia dentaria donde evalúa la población normal y afectada por LPF, demuestra una prevalencia del 0.15 al $16.2 \%$ para la población no afectada (normal) mientras que para pacientes con fisuras, demuestra que la prevalencia fue mucho mayor, alcanzando totales del $29.5 \%$ al $77 \%$ ".

La agenesia se puede manifestar tanto en la dentición temporal como en la permanente y se observa tanto fuera como dentro de la fisura, sin embargo, los valores más altos de agenesia se encuentran en la región de la fisura, además se menciona que la frecuencia de dientes perdidos es considerablemente más alta en la dentición permanente. ${ }^{17,20}$

Sanchez M. en $2020^{1}$ menciona "La prevalencia de agenesia en dientes permanentes varía de $1.6 \%$ a $9.6 \%$, mientras que en dentición temporal el intervalo es de $0.5 \%$ a $0.9 \%$; se ha observado que cuando las agenesias se presentan en la dentición temporal puede repercutir en la dentición permanente, sin embargo, hay casos en los que el diente temporal no existe y el permanente puede estar o no presente donde se encuentra el diente temporal y hay agenesia de su sucesor".

\subsection{Dientes supernumerarios en pacientes con LPF}

El diente supernumerario es definido como aquel órgano dental formado en número excesivo o aquel diente adicional en la dentición normal ya sea temporal o permanente. Puede presentarse en cualquier paciente sin ninguna enfermedad o puede estar relacionado con alguna patología como es el caso de los pacientes con LPF, donde la presencia del diente supernumerario es bastante frecuente. La prevalencia de dientes supernumerarios en individuos con LPF se señala en más del $28 \%{ }^{14,20}$

Según Navas M. en $2017^{15}$ en su investigación señala que el $32 \%$ de la población estudiada (pacientes con labio y paladar fisurado), se manifestó dientes supernumerarios.

\section{Discusión}

La literatura reporta una evidente prevalencia mayoritaria de anomalías dentales en pacientes con LPF, que en la población en general, pues es bien sabido que la genética y factores medioambientales juegan un papel importante en dicha manifestación, de acuerdo a diversos autores citados a continuación, la agenesia y dientes supernumerarios (anomalías dentarias de número) son significativamente las más prevalentes en los niños con LPF. ${ }^{1}$ 
Sánchez M. $2019^{1}$ En su estudio en base a una revisión sistemática de la literatura, menciona que las anomalías más frecuentes que se manifiestan en pacientes con LPF, son anomalías dentarias de número (agenesia y diente supernumerario).

Ventura N. $2020^{21}$ en su estudio de casos y controles con un tamaño muestral de 86 pacientes (6-12 años de edad) con LPF realizado en Brasil demostró a la agenesia dental como la anomalía más prevalente en este grupo.

Yezioro R. $2019^{22}$ en su estudio de casos y controles realizado en Colombia, con un tamaño muestral de 210 pacientes (5-12 años de edad) con LPF, demostró una prevalencia de agenesia del 50.9\%; datos concordantes con las investigaciones realizadas por De Stefani A. $2019^{23}$ (Italia), donde mostró una prevalencia de agenesia dental del $68.6 \%$ en un grupo de 160 pacientes con LPF (7-15 años de edad).

Rizell S. $2019^{13}$ en su estudio realizado en Escandinavia y Gran Bretaña simultaneamente, con un tamaño muestral de 425 individuos con LPF (8 años de edad) demostró una prevalencia del $52.5 \%$ para agenesia dental y un $16.9 \%$ para dientes supernumerarios. De igual manera Yezioro R. en $2019^{16}$ mostró una prevalencia significativa para agenesia dental, en su estudio realizado en Colombia-Bogotá en un grupo de 210 pacientes con LPF (5 a 12 años). Así mismo Elhoseiny Y. $2019^{24}$ de acuerdo a su estudio realizado en Egipto en base a estudios de 22 tomografías dentales de pacientes con LPF (8-14 años de edad), demostró una alta prevalencia de agenesia dental.

Germec D. en $2018^{25}$ en su estudio realizado en Turquía señaló que la anomalía dental más prevalente en 88 pacientes con LPF (edad promedio 6,4 años) fue la agenesia y diente supernumerario. Li E. en $2018^{26}$ mencionó resultados similares en su estudio realizado en Singapur con un tamaño muestral de 60 niños con LPF (niños de 13 años de edad), pues señaló una prevalencia del $63.3 \%$ para agenesia dental, un $21.7 \%$ para dientes supernumerarios.

Menezes C. en $2018^{18}$ mencionó una prevalencia que coincide con los datos anteriormente citados donde destacó una prevalencia del $62 \%$ para agenesia dental, seguida por dientes supernumerarios con una prevalencia del $23.7 \%$, y un $17.8 \%$ los dientes retenidos, estudio retrospectivo en base a radiografías dentales panorámicas con un tamaño muestral de 524 radiografías de pacientes con LPF (menores a 18 años), procedentes de Brasil.

Konstantonis D. en $2017^{27}$ en su estudio retrospectivo realizado en Grecia en base a la observación de radiografías dentales panorámicas, de 154 pacientes con LPF (8 años de edad), señaló una prevalencia del 50\% para agenesia dental. Mangione F. en $2017^{28}$ por su parte señaló una prevalencia del $83.8 \%$ para agenesia dental y un $8.1 \%$ para diente supernumerario, este estudio fue retrospectivo realizado en Francia en un grupo de 74 pacientes con labio y paladar fisurado (6-16 años de edad).

López A. en $2017^{29}$ por su parte señala una prevalencia similar a las antes mencionadas, demostrando una incidencia del $51.2 \%$ para agenesia dental, este estudio se realizó con un tamaño muestral de 118 individuos (10-15 años de edad) con Fisura Labio Palatina procedentes de España. Ajami S. en $2017^{20}$ en su estudio transversal donde incluyó 80 pacientes (8-32 años de edad) con fisura labio palatina demostró una prevalencia significativa para agenesia dental, donde señaló una incidencia del $71.2 \%$ y dientes supernumerarios del $15 \%$, estudio realizado en una población de Irán.

Smalen A. en $2017^{19}$ en un estudio retrospectivo donde evaluó radiografías dentales panorámicas, tanto de pacientes afectados con LPF y pacientes afectados con secuencia de Robin no sindrómica (patología que se caracteriza por la ausencia del cierre del paladar blando debido a la deficiente desarrollo de la mandíbula), encontró una prevalencia del $29.8 \%$ y $47.8 \%$ para agenesia dental respectivamente.

Saele P. en $2016^{30}$ de acuerdo a su estudio realizado en Noruega en pacientes con labio y paladar fisurado de 6 años de edad, demostró una prevalencia del $36.5 \%$, y $17.8 \%$ para agenesia, y dientes supernumerarios, respectivamente, donde el tamaño de muestra fue de 989 pacientes. Sá J. en $2016^{31}$ por su parte señaló una prevalencia del $47.1 \%$ para agenesia dental; datos similares a los encontrados por Saele P., el tamaño de muestra utilizada en este estudio fue de 178 individuos con LPF (menores a 12 años de edad). Por otro lado, Berniczei A. en $2016^{17}$ demostró una prevalencia aún mayor para agenesia dental donde señaló una incidencia del $69 \%$ de 150 pacientes con LPF (6 años de edad), estudio realizado en Hungría.

Vigueras O. en $2015^{7}$ mencionó una prevalencia del $15.4 \%$ para dientes supernumerarios en una población mexicana, con un tamaño muestral de 608 individuos con LPF (2-12 años de edad). Renfigo R. en $2016^{32}$ en su estudio realizado en un grupo de pacientes con LPF (5-15 años de edad), procedentes de Colombia demostró una prevalencia del $90 \%$ para agenesia dental, seguida por dientes supernumerarios en un $40 \%$.

Sá J. en $2015^{33}$ coincidió con varios autores citados, demostró una incidencia de agenesia dental en un $29.2 \%$, seguida de dientes supernumerarios en un $2.6 \%$, siendo estas dos anomalías las más frecuentes encontradas, este estudio fue realizado en pacientes con labio y paladar fisurado de 12 a 45 años de edad. Según Celikoglu M. en 
$2015^{34}$ encuentró una prevalencia bastante significativa para agenesia dental del $92 \%$ en una muestra de individuos de Brasil conformada por 50 participantes con FLP (12- 25 años de edad).

\section{Conclusiones}

Los datos mostrados en la presente revisión de literatura permitieron visualizar la alta prevalencia de anomalías dentarias de número (agenesia y diente supernumerario) presentes en pacientes con Labio y Paladar Fisurado, de esta forma brindamos información actualizada a estudiantes y profesionales de la salud, para futuras consultas e investigaciones científicas.

De acuerdo a la discución realizada la agenesia ocupa la anomalía dental más prevalente en pacientes con Labio y Paladar Fisurado, seguida del diente supernumerario, estas dos anomalías de acuerdo a diferentes estudios originales ocupan los primeros puestos en cuanto a prevalencia.

El profesional debe tener en cuenta dichas alteraciones con el propósito de tomar decisiones acertadas con respecto al manejo integral del paciente, basándose en evidencia científica que respalde cada uno de sus diagnósticos y procedimientos terapéuticos, con el único fin de mejorar la calidad de vida.

Conflicto de intereses: Los autores no manifiestan ningún conflicto de interés.

\section{Referencias}

1. Sánchez Peña M, Galvis Aricapa J.Anomalías dentales de los pacientes con labio y paladar hendido: revisión de la literatura. Rev. Nac. de Odnt. [Internet].2020 Dic.;[Citado 2020 Nov.]; 15(29):1-17.Disponible en: https://revistas.ucc.edu.co/index.php/od/article/view/3343/2947

2. Cazar M,Campos L,Pineda D,Guillén Panorama epidemiológico de la fisura labiopalatina en Quito, Guayaquil y Cuenca. Ecuador, 2010-2018.Acta Odont Col. [Internet].2020; [Citado 2020 Nov.];10(1):37-46. Disponible en: https://revistas.unal.edu.co/index.php/actaodontocol/article/view/82122

3. Palmero J,Rodríguez M.Labio y paladar hendido.Conceptos actuales.Act. Med. Angeles.[Internet].2019;[Citado 2020 Nov.]; 17 (4): 372-379.Disponible en: https://www.medigraphic.com/pdfs/actmed/am-2019/am194j.pdf

4. Martínez L, Imbert Y, Simons S, Herrera Y, Nápoles Y. Combinación de la técnica funcional con Ortodoncia en el tratamiento de pacientes fisurados labio-palatinos. Rev. Inf. Cient. [Internet].2018;[Citado 2020 Nov.]; 97(2):408-420.Disponible en: http://www.revinfcientifica.sld.cu/index.php/ric/article/view/1897/3651

5. Palacios $\mathrm{D}$, Alvear $\mathrm{M}$, Alvarado $\mathrm{A}$, Reinoso S. Atención integral del paciente con labio y paladar fisurado. OActiva UC. [Internet].2020; [Citado 2020 Nov.]; 5(2): 27-30.Disponible en: https://oactiva.ucacue.edu.ec/index.php/oactiva/article/view/462/595

6. Leiva N, Stange C, Ayala F,Fuentes V. Clasificación anatómica: una propuesta para categorizar las fisuras labiopalatinas. Odontol. Sanmarquina.2018; [Citado 2020 Nov.]; 22(3): 245-249.Dsiponible en: https://revistasinvestigacion.unmsm.edu.pe/index.php/odont/article/view/16717/14275

7. Vigueras $\mathrm{O}$, Fernández M, Villanueva M. Prevalencia de dientes supernumerarios en niños con labio y/o paladar fisurado. Rev. Odnt. Mex. [Internet].2015;[Citado 2020 Nov.]; 19(2):81-82.Disponible en: ht t ps://ww w. medigraphic.com/pdfs / odon/uo-2015/uo152c.pdf

8. Jaimes M. Etiología y necesidades pediátricas del paciente con labio paladar y fisurado. Diagnóstico. Diagnostico. [Internet].2018; [Citado 2020 Nov.]; 57(1):25-26.Disponible en: http://142.44.242.51/index.php/diagnostico/article/view/80

9. Reinoso S, Torrachi A, Villavicencio E. Determinantes sociales adversos y Riesgo de Labio y Paladar fisurados. Rev. Fac. de Cien. Med. de la Univ. de Cuenca. [Internet].2020; [Citado 2020 Nov.]; 38(2):1-8.Disponible en: https://publicaciones.ucuenca.edu.ec/ojs/index.php/medicina/article/view/3334/2410

10. Méndez R, García M. Fisuras Labiopalatinas. Actualización en Otorrinolaringología Pediátrica.España: Sociedad Gallega de Otorrinolaringología,2017:57-76.

11. Navarrete E, Canún S, Valdés J, Reyes A. Prevalencia de labio hendido con o sin paladar hendido en recién nacidos vivos. México, 2008-2014. Rev. Mex. Ped. [Internet].2017;[Citado 2020 Nov.]; 84(3):101-110.Disponible en: https://www.medigraphic.com/pdfs/pediat/sp-2017/sp173d.pdf

12. González A, Luna F, Sotelo M, Hernández M, Díaz C. Estudio retrospectivo de la prevalencia de labio y paladar hendido en el Hospital de la Mujer Zacatecana de 2013 a 2015.Rev. Lat. Ort. y Ped. [Internet].2020;[Citado 2020 Nov.].Disponible en: https://www.ortodoncia.ws/publicaciones/2020/art-42/ 
13. Rizell S, Bellardie H, Karsten A, Sæle P, Mooney J, Heliövaara A, et al. Scandcleft randomized trials of primary surgery for unilateral cleft lip and palate: dental anomalies in 8-year olds. Eur J Orthod. [Internet].2019;[Citado 2020 Nov.]; 1-7.Disponible en: https://pubmed.ncbi.nlm.nih.gov/33367600/

14. Rengifo H. Caracterización dental de niños colombianos con hendiduras labio palatinas no sindrómicas. Rev. Odnt. Mex. [Internet].2016; [Citado 2020 Nov.]; 20(3): 179-186.Disponible en: https://www.elsevier.es/es-revista-revista-odontologica-mexicana-90-pdf-S1870199 X16300313

15. Navas M. Prevalencia de dientes supernumerarios en pacientes con labio y paladar hendido mediante el análisis de ortopantomografías. Rev. Esp. Cir. Oral Maxilofac. [Internet].2017;[Citado 2020 Nov.]; 39(3): 171-178.Disponible en: http://scielo.isciii.es/pdf/maxi/v39n3/1130-0558-maxi-39-03-00171.pdf

16. Yezioro S,Rengifo H, Aguilar D. Anomalías dentales en hemiarcada no afectada de sujetos con labio y paladar hendido unilateral no sindrómico. Rev. Mex. Ortod. [Internet].2019;[Citado 2020 Nov.]; 7(2): 57-65.Disponible en: https://www.medigraphic.com/pdfs/ortodoncia/mo-2019/mo192b.pdf

17. Berniczei-Roykó A, Jan-Hendrik T, Krinner A, Gredes T, Végh A, GáborK, et al. Radiographic Study of the Prevalence and Distribution of Hypodontia Associated with Unilateral and Bilateral Clef Lip and Palate in a Hungarian Population. Med Sci Monit. [Internet].2016; [Citado 2020 Nov.]; 22: 3868-3885.Disponible en: https://www.medscimonit.com/abstract/index/idArt/897957

18. Menezes C, De Arruda J, Silva L, Monteiro J, Caribé $P$, Álvares P, et. al. Nonsyndromic cleft lip and/or palate: A multicenter study of the dental anomalies involved. J Clin Exp Dent. [Internet].2018;[Citado 2020 Nov.]; 10(8): 746-750. Disponible en: https://www.ncbi.nlm.nih.gov/pmc/articles/PMC6174020/

19. De Smalen A, Van Nunen D, Hermus R, Ongkosuwito E, Van Wijk A, Don Griot J, et. al. Permanent tooth agenesis in non-syndromic Robin sequence and cleft palate: prevalence and patterns. Clin Oral Invest. [Internet].2017; [Citado 2020 Nov.]; 21:2273-2281. Disponible en: https://www.ncbi.nlm.nih.gov/pmc/articles/PMC5559566/pdf/784_2016_Article_2020.pdf

20. Ajami S, Pakshir H, Samady H. Prevalence and Characteristics of Developmental Dental Anomalies in Iranian Orofacial Cleft Patients. J Dent Shiraz Univ Med Sci.
[Internet].2017;[Citado 2020 Nov.]; 18 (3): 193-200. Disponible en: https://www.ncbi.nlm.nih.gov/pmc/articles/PMC5634359/pdf/JDS-18-193.pdf

21. Ventura N, Assis R, Della R, Carrinho A. Patterns of dental anomalies in patients with nonsyndromic oral cleft.BJOS. [Internet].2020;[Citado 2020 Nov.]; 19 : 1-9. Disponible en: https://periodicos.sbu.unicamp.br/ojs/index.php/bjos/article/view/8658729/22942

22. Yezioro S, Eslava J, Otero L, DDS3, Rodríguez S, Duque A, Campos F. Et.al. The Cleft Palate-Craniofacial Journal. [Internet].2019; [Citado 2020 Nov.]; 20 (10) : 1-7. Disponible en: https://journals.sagepub.com/doi/10.1177/1055665619861498

23. De Stefani A, Bruno G, Balasso P, Mazzoleni S, Baciliero U, Gracco A. Prevalence of Hypodontia in Unilateral and Bilateral Cleft Lip and Palate Patients Inside and Outside Cleft Area: A Case-Control Study. J Clin Pediatr Dent. [Internet].2019; [Citado 2020 Nov.]; 43 (2) : 126-130. Disponible en: https://meridian.allenpress.com/jcpd/article-abstract/43/2/126/9791/Prevalence-of-Hypodontia-in-Unilater al-and?redirectedFrom $=$ fulltext

24. Elhoseiny Y, Mostafa R, Elkassaby M, Abou-El-Fotouh M. Tooth Agenesis in Patients with Complete Bilateral Cleft Lip and Palate. Ann Maxillofac Surg. [Internet].2019;[Citado 2020 Nov.]; 9 (2) : 359-63. Disponible en: cles/PMC6933966/ https://www.ncbi.nlm.nih.gov/pmc/arti-

25. Germec D, Rahime N, Nur F, Ayca A. Dental Anomalies in Different Types of Cleft Lip and Palate: Is There Any Relation?. Journal of Craniofacial Surg. [Internet].2018;[Citado 2020 Nov.]; 29 (5) : 1316-1321. Disponible en: https://journals.lww.com/jcraniofacialsur$\begin{array}{llllllllllllll}\mathrm{g} & \mathrm{e} & \mathrm{r} & \mathrm{y} & / & \mathrm{A} & \mathrm{b} & \mathrm{s} & \mathrm{t} & \mathrm{r} & \mathrm{a} & \mathrm{c} & -\end{array}$ t/2018/07000/Dental_Anomalies_in_Different_Types of_Cleft_Lip.47.aspx

26. Tan E, Kuek M, Wong H, Ong S, Yow M. Secondary Dentition Characteristics in Children With Nonsyndromic Unilateral Cleft Lip and Palate: A Retrospective Study. The Cleft Palate-Craniofacial Journal. Internet].2018; [Citado 2020 Nov.]; 55 (4): 582-589. Disponible en: https://doi.org/10.1177/1055665617750489

27. Konstantonis D, Alexandropoulos A, Konstantoni N, Nassika M. A cross-sectional analysis of the prevalence of tooth agenesis and structural dental anomalies in association with cleft type in non-syndromic oral cleft patients. Prog Orthod. [Internet].2017; [Citado 2020 Nov.]; 18 (20): 1-9. Disponible en: https://progressinor- 
thodontics.springeropen.com/track/pdf/10.1186/s40510-017-0169-x.pdf

28. Mangione F, Nguyen L, Foumou N, Bocquet E, Dursun Aceptado: 29 de abril de 2021 E. Cleft palate with/without cleft lip in French children: radiographic evaluation of prevalence, location and coexistence of dental anomalies inside and outside cleft region. Clin Oral Invest. [Internet].2018;[Citado 2020 Nov.]; 22: 689 - 695. Disponible en: https://link.springer.com/article/10.1007/s00784-017-2141-z

29. López A, Silvestre J, Javier F, Paredes V. Tooth agenesis code (TAC) in complete unilateral and bilateral cleft lip and palate patients. Odontology. [Internet].2017;[Citado 2020 Nov.]; 106: 257-265. Disponible en: https://link.springer.com/article/10.1007\%2Fs10266-017-0332-3

30. Sæle $\mathrm{P}$, Østhus E, Ådalen S, Elwalid F, Mustafa N, Mustafa M. Pattern of clefts and dental anomalies in six-yearold children: a retrospective observational study in western Norway. Acta Odontologica Scandinavica. [Internet].2016;[Citado 2020 Nov.]; 75 (2): 100-105. Disponible en: https://www.tandfonline.com/doi/abs/10.1 080/00016357.2016.1260770? journalCode=iode20

31. Sá J , Araújo L, Guimarães L, Maranhão S, Lopes G, Medrado A, et. al. Dental anomalies inside the cleft region in individuals with nonsyndromic cleft lip with or without cleft palate. Med Oral Patol Oral Cir Bucal. [Internet].2016;[Citado 2020 Nov.]; 21 (1): 48-52. Disponible en: http://www.medicinaoral.com/pubmed/medoralv21_i1_p48.pdf

32. Rengifo H. Caracterización dental de niños colombianos con hendiduras labio palatinas no sindrómicas. Rev Odot Mex. [Internet].2016; [Citado 2020 Nov.]; 20 (3): 179-186. Disponible en: https://www.medigraphic.com/pdfs/odon/uo-2016/uo163e.pdf

33. Sa J, Mariano L, Cangu D, Coutinho T, Hoshi R, Peixoto A. Dental Anomalies in a Brazilian Cleft Population. The Cleft Palate-Craniofacial Journal. [Internet].2016; [Citado 2020 Nov.]; 53 (6): 724-729. Disponible en: https://journals.sagepub.com/doi/10.1597/14-303?url_ver $=$ Z39.88-2003\&rfr_ id=ori:rid:crossref.org\&rfr_da$\mathrm{t}=$ cr_pub $\% 20 \% 200$ pubmed

34. Celikoglu M, Buyuk S, Sekerci A, Antekin K, Candirli C. Maxillary Dental Anomalies in Patients with Cleft Lip and Palate: A Cone Beam Computed Tomography Study. J Clin Ped Dent. [Internet].2015;[Citado 2020 Nov.]; 39 (2): 183-186. Disponible en: https://doi.org/10.17796/jcpd.39.2.t623u7495h07522r 